\title{
PLANIFICACIÓN DEL PROCESO ENFRIAMIENTO EN EL ALMACENAMIENTO DE MAÍZ EN SILOS METÁLICOS REFRIGERADOS
}

\begin{abstract}
Esta investigación tiene como objetivo pronosticar el comportamiento de la temperatura en un Silo Metálico Refrigerado (SMR) para realizar una planificación del proceso de enfriamiento eficaz. Este proceso se realiza a través de la conexión a los SMR de compresores de aire frío acoplados a vehículos, los cuales son compartidos entre varios silos en la región. Se estudiaron estadísticamente las variables del clima durante el almacenamiento del maíz utilizando el análisis de capacidad, lo que arrojó como resultados que la temperatura no cumplía con las especificaciones lo que provoca la aparición de plagas durante el período poscosecha y con ello el deterioro del grano. Se construyó un modelo de predicción utilizando las líneas de tendencia y se realizó un análisis de errores para validar el modelo. Como resultado se utilizó el modelo de predicción de la temperatura y se planificó el proceso de enfriamiento que garantiza el control de la temperatura en los SMR.
\end{abstract}

* Máster en Ingeniería Industrial mención Logística, Profesor auxiliar, Departamento de Ingeniería Industrial, Universidad de Cienfuegos, Provincia de Cienfuegos, Cuba (e-mail: mfeito@ucf.edu.cu). 


\section{INTRODUCCIÓN}

Ainicios del milenio la población mundial ha aumentado considerablemente, las necesidades alimentarías han sido mayores de acuerdo con el aumento demográfico y en cambio, la producción de los productos y subproductos para la alimentación no ha crecido de acuerdo con el crecimiento poblacional. Según la estimación de la FAO, en el futuro la demanda de los alimentos aumentará un $3 \%$ por año, sin embargo la producción alimentaría únicamente tendrá un aumento del 2,8 \% por año, unido a la fabricación de biocombustibles (LANDIS, 2008). Por lo que el almacenamiento y conservación de los productos y subproductos (granos, cereales, frutas, hortalizas, conservas, productos industrializados, etc.) para la alimentación humana y animal requieren de mayor atención con el fin de solventar todas las necesidades.

La cadena del maíz ocupa un lugar prioritario en el desarrollo de casi todos los países del mundo debido a su capacidad de generación de empleo, inversión, desarrollo regional y a las innumerables oportunidades de crecimiento y progreso que ofrece (BERRY, ROBERTS y SCHLENKER, 2012). Este fenómeno se observa tanto en los países que lo producen en gran cantidad, como Brasil, los Estados Unidos o la Unión Europea; como en aquellos que deben importarlo para abastecer sus industrias como Japón o Corea.

El cultivo del maíz en el continente americano representa casi el $40 \%$ del área ocupada en el mundo por este cultivo. En América del Norte, los Estados Unidos son el centro productor, con más del $45 \%$ de la producción mundial y constituye el cultivo más importante, utilizando para la alimentación humana y como alimento básico de las dietas para animales (PALIWAL et al., 2001). Dentro de Latinoamérica, todos los países cultivan este cereal, entre ellos se destacan, Brasil, México y Argentina. Europa ocupa el segundo lugar en cuanto a producción y sobresalen Francia, Rumania y Yugoslavia. En este contexto, se describen diversos factores, bióticos y abióticos, que son los causantes de pérdidas en la producción y almacenamiento de maíz a nivel mundial como se muestran en la Figura 1 (PINGALI, 2001). Factor abiótico es, principalmente, la infertilidad del suelo y los insectos poscosecha como factor biótico; en conjunto estos factores son responsables de pérdidas entre 70 a $95 \%$ de la cosecha cuando las condiciones de cultivo y almacenamiento son deficientes. Las plagas causan pérdidas superiores al $10 \%$ durante la producción y de 10 a $20 \%$ en poscosecha.

Las principales plagas que afectan el grano durante su almacenamiento son el gorgojo de maíz, Sitophilus zeamais, en las regiones tropicales y subtropicales; el barrenador grande del grano, Prostephanus truncatus, localizado en las regiones de transición y altas y la palomilla dorada de maíz, Sitotroga cerealella, ubicada en las regiones templadas y altas. Plagas secundarias que aparecen con menor frecuencia son: Plodia interpuctella, Tribolium castaneum, Cathartus quadricollis, Rhyzoperta dominica, Cryptolestes ferrugineus, entre otras (DOMÍNGUEZ UMPIÉRREZ y MARRERO ARTABE, 2010).

En Cuba debido a la especialización en el cultivo de la caña de azúcar, arroz, viandas y cítricos la producción de otros granos y cereales como maíz y leguminosas es escasa por lo que se importan grandes cantidades para garantizar el consumo humano y animal. Es por esto que la conservación de los mismos en óptimas condiciones reviste mayor importancia. Las condiciones tropicales imperantes en Cuba, favorecen grandemente la proliferación de las plagas dentro de los almacenes (ACOSTA, 2012; PALIWAL et al., 2001; YANG et al., 2012), debido a que estas requieren valores elevados de temperatura para su desarrollo, reproducción y mantenimiento en el ambiente.

La finalidad principal del almacenamiento está en guardar parte o la totalidad de las cosechas o subproductos elaborados en correspondencia con necesidades tales como: contar con semillas certificadas para los cultivos de las siguientes temporadas, constituir una reserva de alimentos para épocas posteriores, esperar mejores precios en los mercados locales e internacionales y utilizar los productos de acuerdo con las necesidades de consumo, industriales y de mercado (RUIZ 
ARBOLEDA, 2010). La conservación de los productos agropecuarios es necesaria para lograr su disponibilidad en un momento dado a lo largo de todo un período. Durante la conservación el producto puede mantener sus cualidades originales o sufrir transformaciones, según el proceso y los objetivos.

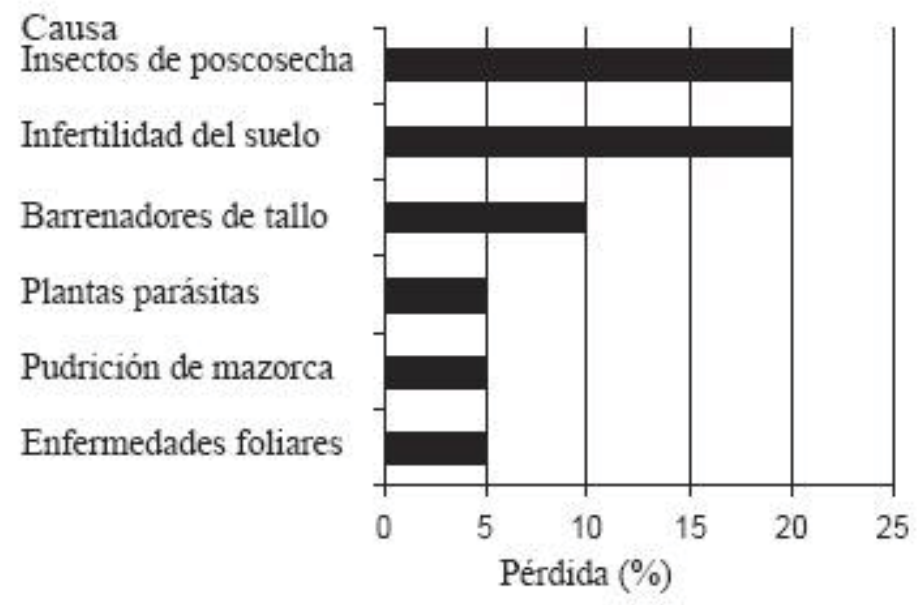

\section{FIGURA 1 - PRINCIPALES FACTORES QUE PROVOCAN PÉRDIDAS EN LA PRODUCCIÓN Y ALMACENAMIENTO DE MAÍZ (PINGALI, 2001)}

En general, los granos deben almacenarse para mantenerlos físicamente bien y conservar su poder germinativo. Los granos pueden deteriorarse físicamente por la acción de bacterias y hongos, por lo se debe tener en cuenta en las condiciones de almacenamiento. El principio más usado en esta etapa para la prolongación marcada de la vida útil de los productos agropecuarios es el del almacenamiento refrigerado o de baja temperatura bajo condiciones controladas de humedad y temperatura, con lo que se reduce la actividad biológica del producto y de los microorganismos, y en el caso de granos y especias se evita la incubación de los huevos de insectos (CHUNHUA, 2011; HUI; LIWEI y JIAMIN, 2010; HUI, 2010; LEI, 2011).

El almacenamiento de granos, constituye una actividad muy especializada que requiere de tecnologías apropiadas, para garantizar su conservación, con la calidad necesaria como uso industrial o consumo directo. Partiendo de lo anterior, el principio para lograr que el almacenamiento de los granos se efectúe correctamente, radica en que estos deben conservarse limpios, sanos, secos y fríos y en un lugar o depósito que pueda mantener las temperaturas y las condiciones iniciales de calidad hasta que el producto llegue a su destino final. Los métodos de almacenamiento en las diferentes regiones del mundo van a estar en dependencia de las necesidades de cada lugar, las condiciones económicas, así como del objetivo del almacenamiento.

Para conservar productos en su estado natural con el mínimo de cambios y pérdidas, es necesario controlar: temperatura, humedad relativa, condiciones del local. La humedad y las altas temperaturas combinadas propician, en primer lugar, las actividades biológicas de los tejidos vegetales (respiración principalmente), hasta la germinación o brote, y también el desarrollo de enfermedades que puedan estar en el producto o que se provoquen por accidentes. A temperaturas y humedad relativa bajas, los efectos anteriores disminuyen o cesan, incluido el ataque de insectos. CHUNHUA, 2011; DOMINGUEZ-PACHECO et al., 2012; HUI, LIWEI y JIAMIN, 2010; HUI, 2010; LADINO GAIBOR, 2012; LEI, 2011; PALIWAL et al., 2001; PINGALI, 2001; RUIZ ARBOLEDA, 2010 señalan que las bajas temperaturas tienen acción inhibidora en las reacciones bioquímicas de la germinación y de la fermentación así como en la supervivencia de agentes bióticos destructores de los granos. 
Las plagas que afectan el maíz requieren temperaturas sobre $14^{\circ} \mathrm{C}$ para poder multiplicarse. Debajo de esta temperatura muchas plagas mueren lentamente, los huevos, larvas y pupas a menudo son susceptibles a las temperaturas ligeramente bajas (CNSV, 2006). El almacenamiento a bajas temperaturas y bajo las condiciones secas es una herramienta de manejo útil para llevar la infestación a un bajo nivel. La humedad por encima de 65-70\% acelera el desarrollo de mohos y a temperaturas entre 20 y $35^{\circ} \mathrm{C}$ se observa el crecimiento óptimo de microorganismos (mohos) y de insectos.

El desarrollo de tecnologías avanzadas como la conservación de los granos en almacenes y sobre todo en dispositivos de mayores requerimientos técnicos como los Silos Metálicos Refrigerados (SMR) ha logrado que las producciones se conserven por mayor espacio de tiempo, en condiciones más homogéneas, libres de cambios bioquímicos y del daño ocasionado por las plagas. Dentro de los silos, se debe mantener un valor constante de temperatura y humedad para no favorecer los procesos fermentativos que pueden sufrir los granos en condiciones de almacenamiento. El aislamiento total con el exterior del producto almacenado y su consecuente conservación en el tiempo es la premisa fundamental de este tipo de sistema de almacenaje (WEIZHI, 2010).

La variable temperatura es la más importante en el proceso de conservación de los granos en los Silos Metálicos Refrigerados (SMR), pues esta influye en el tiempo de almacenamiento de los granos, además en la velocidad de desarrollo de hongos y plagas y solo logramos controlarla con un buen enfriamiento, entonces partiremos de la importancia o finalidad de la refrigeración del grano.

La refrigeración de los SMR en Cuba se realiza a través de la inyección de aire seco a baja temperatura mediante el uso de las máquinas enfriadoras CoolSeed. Este constituye también un método de secado, lo cual se debe lograr con un costo razonable en tiempo y recursos. Esta tecnología se encuentra instalada en medios de transporte automotores lo que permite su utilización y enfriamiento de varios silos. El uso eficiente de los carros de frío depende en gran medida de una correcta planificación y distribución entre los diferentes SMR para evitar que la temperatura de los mismos sobrepase los límites de $20^{\circ} \mathrm{C}$.

Esta investigación tiene como objetivo analizar el comportamiento de la temperatura y la humedad en el SMR dedicado a la conservación de maíz, para planificar más eficaz y eficientemente el proceso de enfriamiento y disminuir las pérdidas por infección de plagas.

\section{MATERIALES Y MÉTODOS}

\subsection{SELECCIÓN DEL OBJETO DE ESTUDIO}

Debido a que el objetivo de esta investigación es el análisis del comportamiento de los factores atmosféricos que influyen en las pérdidas por plagas en el almacenamiento del maíz en Cuba se toman valores de la posición de silo Guillermo Moncada de ubicada en la provincia de Cienfuegos, en los meses enero/marzo de 2013. La selección se debe a que este es el de mayor capacidad en el territorio, se comenzó el almacenamiento el 1ero de enero y además ha mantenido afectaciones por plagas en el año 2012.

\subsection{OBTENCIÓN DE LOS DATOS}

Durante el almacenamiento se toma una lectura diaria (8 de la mañana) la cual indica la temperatura que poseen los sensores ubicados en los 6 cables, por lo que se suman la temperatura de cada cable y se divide entre los sensores del mismo obteniéndose el promedio por cable. De igual forma se promedian las mediciones de todos los sensores y se obtiene el promedio general de temperatura en el interior del silo.

La medición y control de la temperatura de los granos se efectúa con un equipo TermoColector Grupo FOCKINK que desarrolla un sistema de sensores. Este Termo-colector posee una batería recargable la cual no debe tener menos de $90 \%$ de carga si se desea lograr una lectura 
confiable el mismo es conectado a una caja de termometría la cual recibe la información de todos los sensores que se encuentran en el interior del silo. Además este equipo brinda la medición de la temperatura ambiente y la posibilidad de descargar las lecturas directamente a la computadora mediante software que realiza los cálculos y muestra el comportamiento de las temperaturas en cada sensor mediante gráficos.

El valor de humedad que se obtiene a partir del detector rápido de humedad será utilizado para estimar desviaciones marcadas de este parámetro. Como este parámetro se mide a partir de muestras del grano de diferentes partes del SMR solo se realiza una medición semanal, siendo 13 observaciones durante el período analizado.

\subsection{ANÁLISIS ESTADÍSTICO DE LOS DATOS}

Se procesa estadísticamente las variables de temperatura y humedad. Para su procesamiento se prueba primeramente el ajuste a la distribución normal a través de la prueba de Bondad de Ajuste de Kolmogorov-Smirnov. Se realizan análisis de capacidad para las variables temperatura y humedad. Se analiza la independencia estadística utilizando el estadístico ChiCuadrado de Pearson de estas variables para conocer si existe una relación entre las mismas dentro del SMR. Para la realización de las pruebas estadísticas se utiliza el software StatGraphics VI Centurion (STATPOINT TECHNOLOGIES, 2009).

Para la conformación del modelo se propone utilizar las pendientes de las rectas resultante de la regresión lineal y para la decidir si el modelo de predicción propuesto es confiable se calculan las estadísticas de error Media Absolute Desviation (MAD), el cual promedia el valor absoluto de los errores y la señal de rastreo la cual divide la suma de los errores entre el MAD. Estos análisis se realizaron y graficaron utilizando el procesador de hojas de cálculo Microsoft Excel (MICROSOFT CO., 2007).

\section{RESULTADOS Y DISCUSIÓN}

\subsection{JUSTIFICACIÓN DEL ESTUDIO}

La presencia de plagas en las SMR en la provincia Cienfuegos provocó en varias ocasiones la venta del maíz con muy poco tiempo de almacenamiento y debido a la infestación la imposibilidad del consumo humano. En la Tabla 1 se muestran los niveles de infección en los diferentes silos de la provincia siendo los silos de la posición Guillermo Moncada los que tuvieron mayores pérdidas, las causas potenciales el mal uso de los carros de frío lo que provocó altas temperaturas en los mismos.

TABLA 1 - NIVELES DE PLAGA EN LOS SMR PARA ALMACENAMIENTO DE GRANOS EN CIENFUEGOS

\begin{tabular}{|c|c|c|c|c|}
\hline $\begin{array}{c}\text { POSICIÓN DE } \\
\text { SILO }\end{array}$ & TONELADAS & SILO & TIPO DE PLAGA & $\begin{array}{c}\text { NIVEL DE } \\
\text { PLAGAMIENTO }\end{array}$ \\
\hline $\begin{array}{c}\text { Guillermo } \\
\text { Moncada }\end{array}$ & 6000 & $\mathrm{~A}$ & Sitophilus oryzae & Ligero \\
\hline & & Tribolium castaneum & Medio \\
\hline Ramón Balboa & 4000 & $\mathrm{~B}$ & Sitophilus oryzae & Medio \\
\cline { 3 - 5 } & & & Tribolium castaneum & Medio \\
\hline Mal Tiempo & 2000 & $\mathrm{~A}$ & Tribolium castaneum & Ligero \\
\hline
\end{tabular}


Las plagas insectiles poscosecha se desarrollan en un rango óptimo entre $25^{\circ} \mathrm{C}$ y $35^{\circ} \mathrm{C}$. Temperaturas debajo de $20^{\circ} \mathrm{C}$ reducen su tasa de crecimiento a puntos tan bajos que los niveles de daños son casi insignificantes, pero algunos son resistentes como los Sitophilus (CHUNHUA, 2011; HUI, LIWEI y JIAMIN, 2010; LADINO GAIBOR, 2012; LEI, 2011; PALIWAL et al., 2001; PINGALI, 2001; WEI y JIBIN, 2011; WEIZHI, 2010; YANG et al., 2012). Los productos almacenados en los silos no deben tener puntos inferiores a $12{ }^{\circ} \mathrm{C}$ ni mayores a $20^{\circ} \mathrm{C}$, siendo lo óptimo mantener la temperatura de todos los puntos del grano entre estos valores y el promedio de temperatura del silo no debe sobrepasar los $17,5^{\circ} \mathrm{C}$. La práctica en las condiciones actuales indica que en el nivel superior del silo persisten temperaturas por encima de $25^{\circ} \mathrm{C}$, muy favorables al desarrollo de insectos. Según las normas establecidas para un correcto almacenamiento del maíz, la temperatura se debe encontrar entre los $15^{\circ} \mathrm{C}$ y $20^{\circ} \mathrm{C}$. Cuando la temperatura del maíz es alta este queda vulnerable a la aparición de plagas. Si estas son bajas el grano se cristalizaría lo que afectaría su molido, lo que provoca pérdidas y consigo reclamaciones de los clientes.

\subsection{ANÁLISIS DE LA TEMPERATURA EN EL SMR}

Para probar que la capacidad del proceso de mantenimiento de la temperatura en este SMR es deficiente se analiza estadísticamente el comportamiento de esta variable. Para ello se prueba la normalidad de la distribución de la variable utilizando la prueba de bondad de ajuste Kolmogorov-Smirnov. Dado que el menor valor de probabilidad es 0,175971 y es mayor que 0,05, no se puede rechazar la hipótesis nula de que la variable temperatura proviene de una distribución Normal con $95 \%$ de confianza.

Utilizando el software estadístico StatGraphics se determinaron varios índices de capacidad (CHOWDHURY, 2013). Pp es un índice que divide la distancia entre los límites de las especificaciones entre seis veces la desviación estándar, un valor de Pp de 0,89 no es considerado bueno. Ppk es un índice que divide la distancia de la media al límite de especificación más cercano entre tres veces la desviación estándar, en este caso, Ppk es igual a 0,36. La diferencia entre Pp y Ppk demuestra que la variable no está centrada, lo que puede observarse en la Figura 2 así como que existen valores por encima del límite de las especificaciones, mostradas con las líneas largas. Puesto que K es igual a 0,60 , la media está localizada $60 \%$ desde el centro de las especificaciones hacia el límite superior de especificación, lo que puede contribuir de manera significativa a que la capacidad del proceso para cumplir con las especificaciones sea baja. Como el valor de Cp es mayor que 2 se determina que el proceso es adecuado. El índice Cpk es mucho más pequeño que el de $\mathrm{Cp}$, lo que indica que la media del proceso está alejada de las especificaciones.

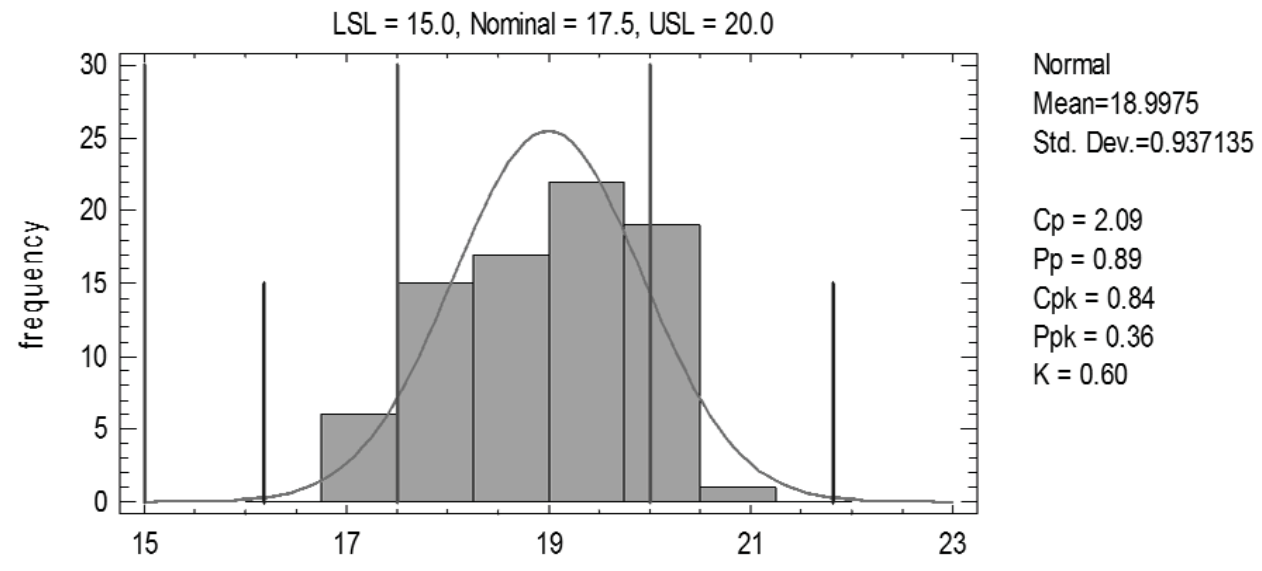

\section{FIGURA 2 - CAPACIDAD DEL PROCESO DE MANTENCIÓN DE LA TEMPERATURA EN EL SILO}




\subsection{ANÁLISIS DE LA HUMEDAD EN EL SMR}

A medida que aumenta la humedad del grano por encima de la humedad de recibo, aumenta el deterioro, principalmente causado por el desarrollo de hongos, levaduras y bacterias. Estos microorganismos necesitan de humedad para crecer y a medida que se van desarrollando, aumentan su nivel de respiración y la temperatura de la masa de los granos.

En general se pueden guardar granos con contenidos de al menos $14 \%$ de humedad en equilibrio con una humedad relativa de menos del $70 \%$ sin peligro de infección por microorganismos. Aún con humedades bastantes bajas, los insectos atacan al grano. Muy pocos insectos atacan los productos almacenados causando deterioro al grano con menos del $10 \%$ de humedad, ya que la mayoría de estos mueren en condiciones de menos del $12 \%$. En el caso del maíz los índices establecidos para mantener su integridad física durante el tiempo de conservación están entre el $10 \%$ y $14 \%$ de humedad.

Para determinar si la distribución Normal es apropiada para estos datos, se realiza la Prueba de Bondad de Ajuste siendo el valor de probabilidad de 0,84 mayor que 0,05 por lo no se puede rechazar la idea de que la humedad proviene de una distribución normal con $95 \%$ de confianza.

La humedad del grano debe de estar entre $10 \%$ y $14 \%$ para que tenga un comportamiento adecuado para la conservación del grano. El análisis de la capacidad de la variable humedad se muestra en la Figura 3.

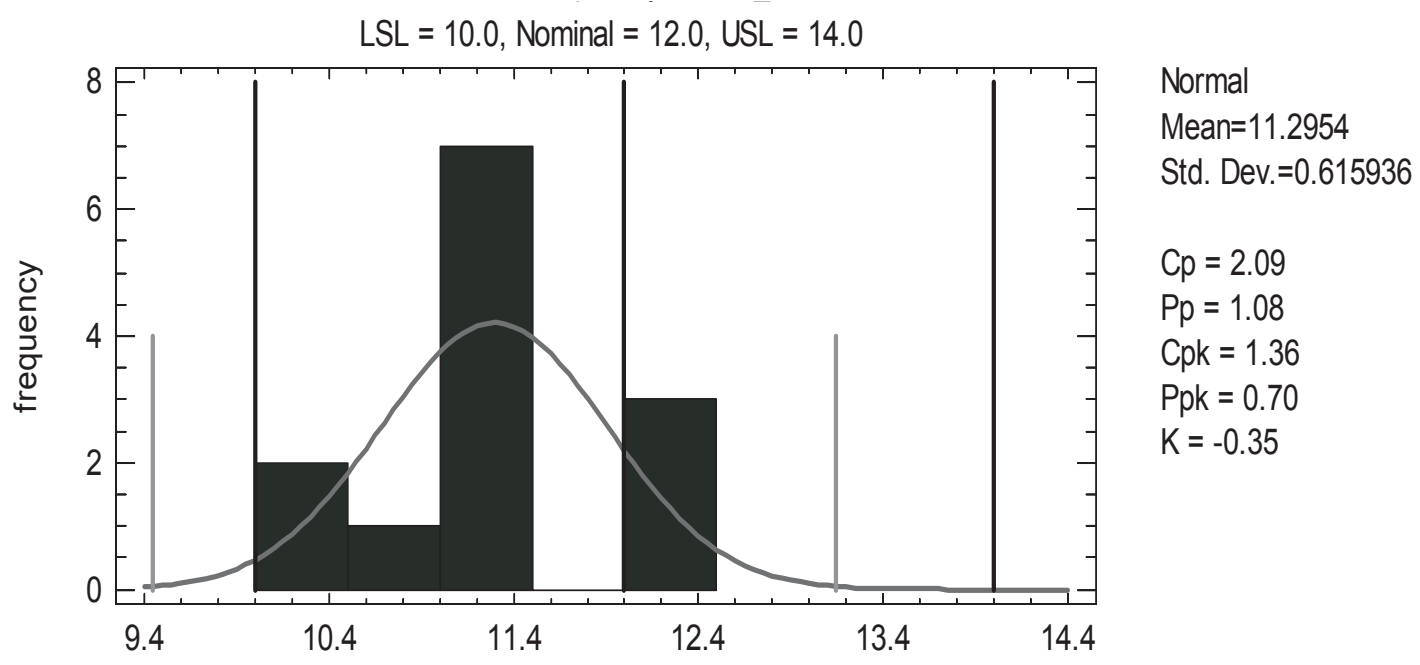

\section{FIGURA 3 - ANÁLISIS DE LA CAPACIDAD DE LA VARIABLE HUMEDAD EN EL SMR}

Se han calculado diversos índices de capacidad para resumir la comparación entre la distribución ajustada y las especificaciones. En este caso, el Pp es igual a 1,08 el cual generalmente se considera que está bien. Puesto que K es igual a $-0,35$, la media está localizada un $35 \%$ desde el centro de las especificaciones hacia el límite inferior de especificación. Como este valor es mayor que el $20 \%$ indica que le proceso está un poco descentrado.

El valor de $\mathrm{Cp}$ es de 1,61 lo que indica que el proceso es adecuado. El índice Cpk es igual a 1,14 en este caso es mucho más pequeño que $\mathrm{Cp}$ establece que la media del proceso está alejada del centro, pero con poca variabilidad. En este caso la media del proceso esta corrida hacia cerca del límite inferior, la poca humedad de los granos lo hace que el maíz pierda peso por la falta de contenido de agua, aunque no en medidas alarmantes. 


\subsection{ANÁLISIS DE INDEPENDENCIA ENTRE LA TEMPERATURA Y \\ LA HUMEDAD}

Con el objetivo de enfocar el estudio y mejorar la capacidad del proceso de almacenamiento del maíz el cual se ve afectado por valores de la temperatura fuera de los parámetros establecidos, se quiere conocer si los cambios para mejorarla no afectan el comportamiento de la humedad. Para ello se realiza la prueba de independencia Chi-cuadrado de Pearson (Tabla 2) para los valores de temperatura en el día que se analizó la humedad (MONTGOMERY, RUNGER y HUBELE, 2009).

\section{TABLA 2 - PRUEBA DE INDEPENDENCIA ESTADÍSTICA ENTRE TEMPERATURA Y HUMEDAD EN EL SMR}

\begin{tabular}{c|c|c|c}
\hline Prueba & Estadístico & Grados de libertad & Probabilidad \\
\hline Chi-Cuadrado de Pearson & 117.000 & 110 & 0,3060 \\
\hline
\end{tabular}

La hipótesis nula de esta prueba es que las variables son independientes entre sí. Según los resultados que muestra la tabla las el valor de probabilidad para la prueba de Chi-cuadrado de Pearson es mayor que a la cual está fijada en 0,05 lo que expresa que las variables temperatura y humedad son independientes con $95 \%$ de confianza. La realización de esta prueba permite efectuar acciones para corregir la temperatura sin temor a afectar la humedad en el proceso de almacenamiento de granos.

\subsection{CONSTRUCCIÓN DE UN MODELO DE PRONÓSTICO DE LA TEMPERATURA EN EL SMR}

Con el objetivo de predecir el comportamiento de la temperatura se grafican los valores de temperatura en función del tiempo (ABRAHAM y LEDOLTER, 2009; BOX, JENKINS y REINSEL, 2013) cuando no está conectado el carro de frío como se muestra en la Figura 4. Aunque el coeficiente de correlación no es alto para ambos casos es visible la tendencia de incremento de la temperatura cuando el carro de frío no está conectado al SMR.
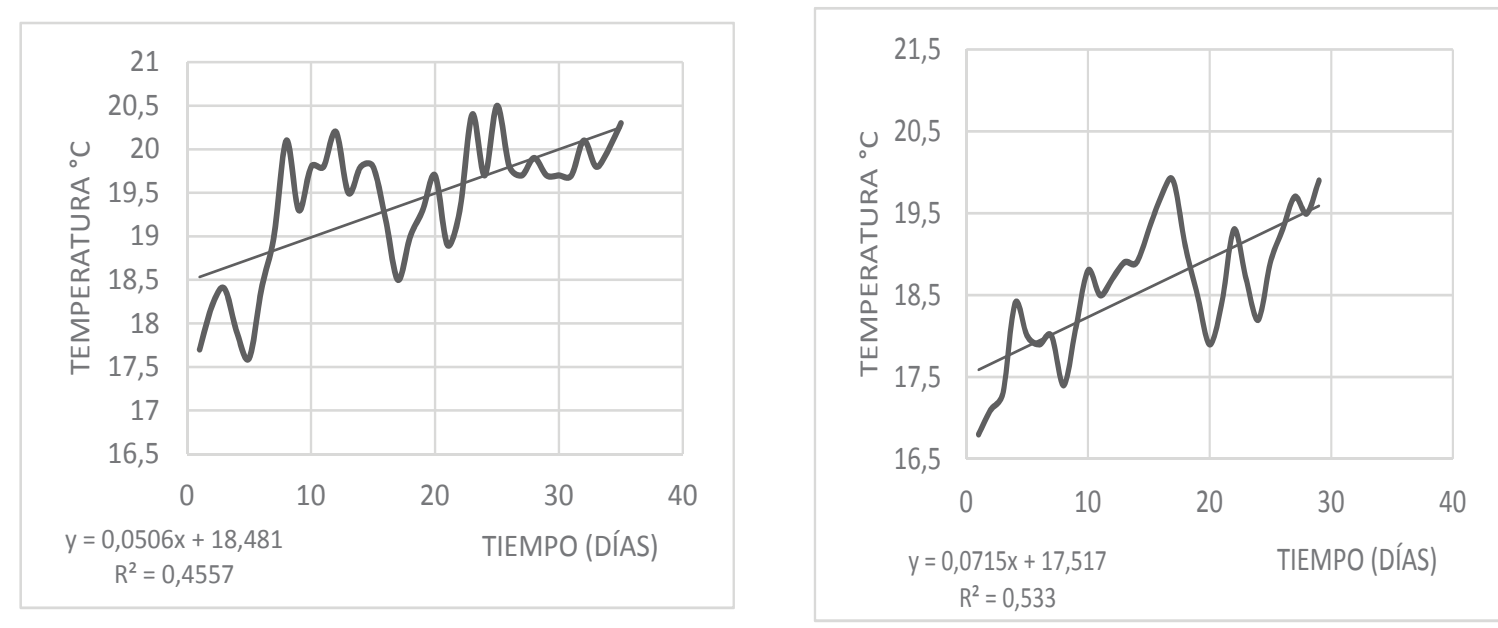

FIGURA 4 - COMPORTAMIENTO DE LA TEMPERATURA CUANDO NO ESTÁ CONECTADO EL CARRO DE FRÍO A) PERÍODO DE 9 DE ENERO - 12 DE FEBRERO 2013

B) PERÍODO DE 21 DE FEBRERO - 20 DE MARZO DE 2013 
Cuando el carro de frío está conectado se puede observar que la línea de tendencia para cada período tiene un coeficiente de correlación muy bueno, es decir que el comportamiento de la temperatura cuando está enfriándose el SMR tiene un comportamiento lineal. En la Figura 5 (a) el carro de frío se conecta al silo 24 horas antes del llenado del arribo del grano por lo que la temperatura baja más rápidamente que cuando es conectado durante el almacenamiento.
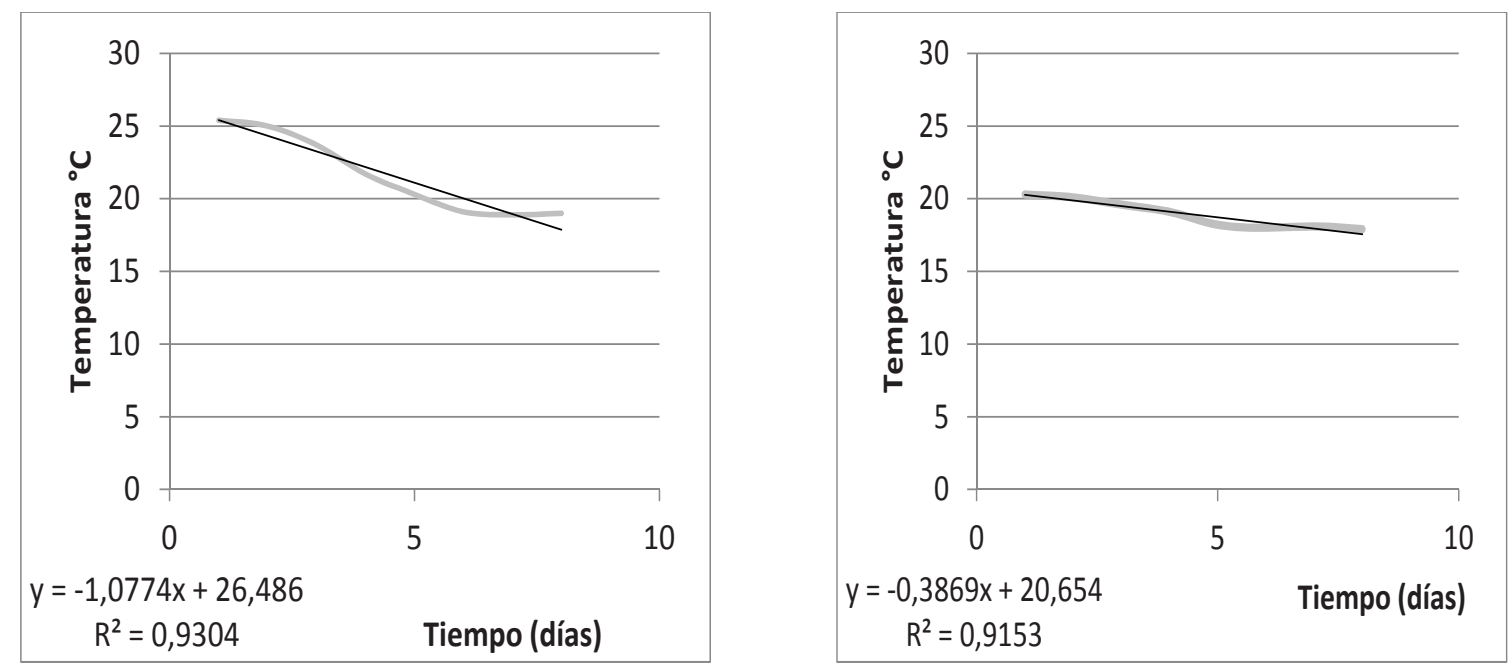

\section{FIGURA 5 - COMPORTAMIENTO DE LA TEMPERATURA CUANDO ESTÁ CONECTADO EL CARRO DE FRÍO A) PERÍODO DE 1 ENERO - 8 ENERO DE 2013 B) PERÍODO DE 13 DE FEBRERO - 20 DE FEBRERO DE 2013}

Para realizar la predicción de los valores de la temperatura cuando entra el primer carro de frío se toma la ecuación de la recta correspondiente a este período, ya que estos datos fueron tomados durante el llenado del silo donde el grano viene con temperaturas muy altas. En el caso de los otros dos carros se toma la pendiente de la recta que más se demora en bajar la temperatura la cual se corresponde con la Figura 5 (b), para pronosticar el peor escenario y cuando no está conectado el carro de frío se calcula con la pendiente de la recta que más rápido sube la temperatura.

Las nuevas rectas de predicción se forman utilizando las pendientes antes explicadas multiplicadas por el valor de "X", sumándole el valor de la temperatura del período anterior. El comportamiento de la predicción y el de los valores reales de la temperatura para la muestra utilizada se muestran en la Figura 6 . El valor de " $X$ " representa los días, cada vez que cambie la ecuación " $X$ " comenzará por el primer valor que sería "1". Se construye el modelo de predicción y se calculan los errores basados en la diferencia de los valores reales con los de la predicción, se calcula la media de las desviaciones absolutas (MAD) y la señal de rastreo que no es más que la división de la suma de los errores entre el MAD (ABRAHAM y LEDOLTER, 2009; KIM, DEKKER y HEIJ, 2013), ésta indica la tendencia del modelo de pronóstico y la separación de las desviaciones.

EI MAD con un valor de 0,737 es muy bueno, debido a que se encuentra cercano a cero. La señal de rastreo de la variable temperatura no es buena ya que el valor de la suma es igual a $-10,973$ y este se debe encontrar entre \pm 6 . Como la desviación es negativa significa que el modelo predice los valores de la temperatura por encima de lo que puede encontrase realmente, este hecho se explica debido a que se está pronosticando el peor escenario. Por dicho motivo aunque la señal de rastreo no está dentro de los parámetros se considera que el modelo de predicción es adecuado para ser utilizado para la planificación de la conexión de los carros de frío en el SMR. 


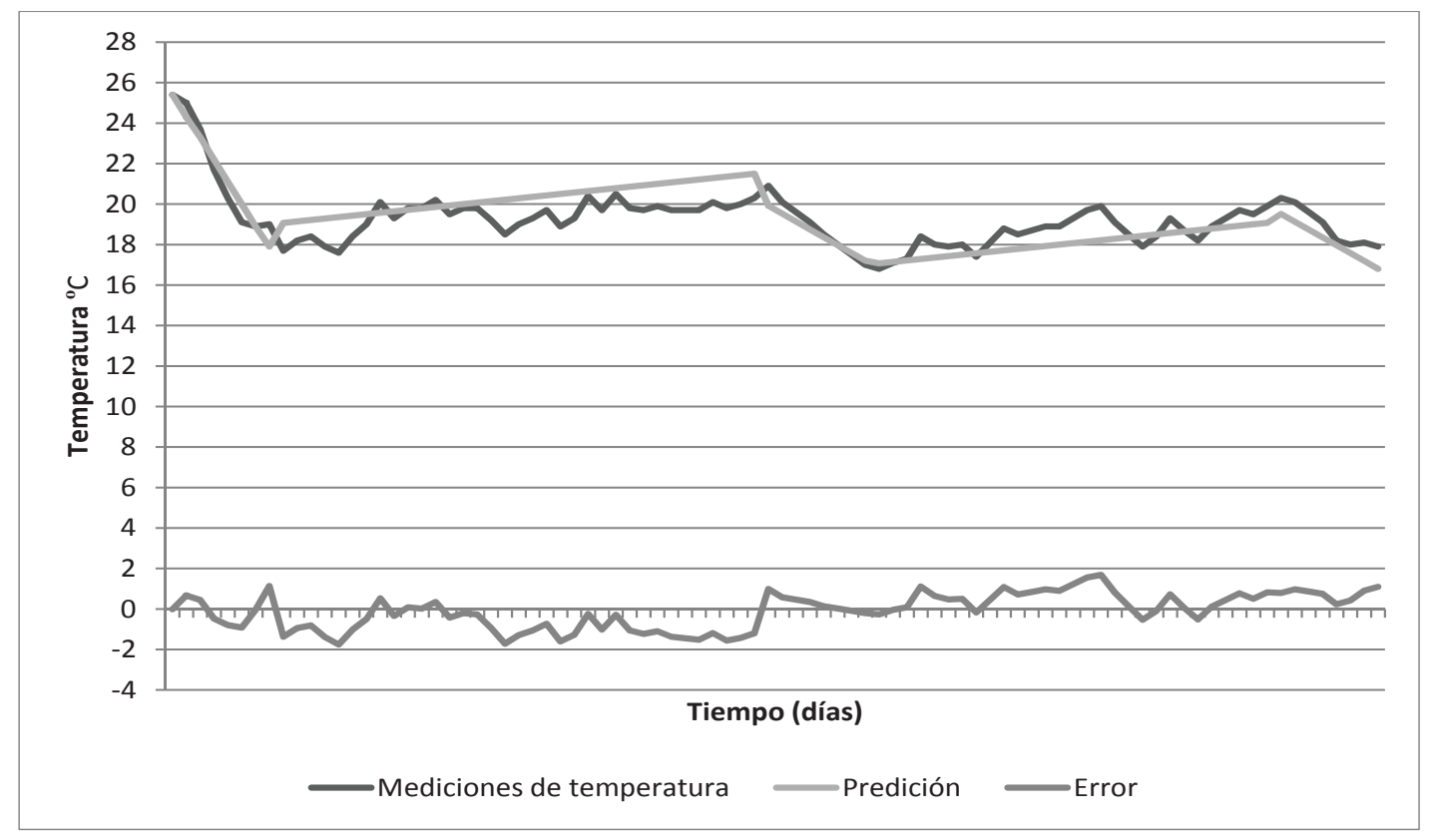

\section{FIGURA 6 - CONSTRUCCIÓN DEL MODELO DE PRONÓSTICO DE LA TEMPERATURA EN LA CONSERVACIÓN DEL MAÍZ EN EL SMR}

\subsection{PLANIFICACIÓN DE LOS CARROS DE FRÍO PARA LA RECEPCIÓN Y CONSERVACIÓN DEL MAÍZ}

Basados en los resultados obtenidos se puede decir que el método predice bastante bien el comportamiento de la variable que es objeto de estudio, tanto en condiciones normales de operación y cuando se encuentran conectados los carros de frío. Seguidamente se procede a planificar los días que se le debe dar enfriamiento al grano que se encuentra almacenado en el silo y los días de antelación en que se deben pedir los carros de frío. Para ello se utiliza el modelo de predicción. En este caso en la ecuación de la recta el valor de la temperatura que se toma es el anterior al de la planificación excepto los primeros valores que se planifica con el valor de la temperatura de la ecuación de la recta que es 26,486 como se muestra en la Figura 7.

Según el procedimiento actual de enfriamiento el carro de frío debe pedirse al proveedor cuando la temperatura del silo se encuentra en $20{ }^{\circ} \mathrm{C}$, demorándose hasta 5 días en dar respuesta y comenzar el proceso de enfriamiento. Una vez conectado se mantiene durante 8 días desconectándose independientemente del valor de la temperatura en el silo.

Como se puede ver en la Figura 6 con la planificación de los carros de frío se mantiene la temperatura dentro de los parámetros establecidos y por debajo de la mayoría de los valores medidos para un período similar. Solo se tienen los primeros valores fuera de los límites de especificaciones durante la recepción del grano. El carro de frío se conecta un día antes de la recepción del maíz y se desconecta a los 10 días cuando la temperatura del silo llega a los $15,71{ }^{\circ} \mathrm{C}$. Se conecta nuevamente cuando la temperatura en el silo llega de $19,54^{\circ} \mathrm{C}$ y se mantiene dando enfriamiento al grano hasta el 20 de marzo, con un valor de la temperatura en el silo de $15,67^{\circ} \mathrm{C}$.

El principal resultado de este plan reside en que se puede notificar con tiempo al proveedor de los carros de frío y este permanece conectado el tiempo necesario a diferencia del método actual. La cantidad de tiempo en que está enfriando el CoolSeed es aproximadamente igual para el período estudiado lo que garantizaría una mejor utilización de la tecnología instalada. Este método puede servir para predecir el comportamiento de la temperatura en el resto de los SMR de la región y realizar una programación del uso y balancear la carga con la disponibilidad de los carros de 
frío. Programar las acciones de mantenimiento de esta tecnología de manera que no afecte el enfriamiento en los SMR.

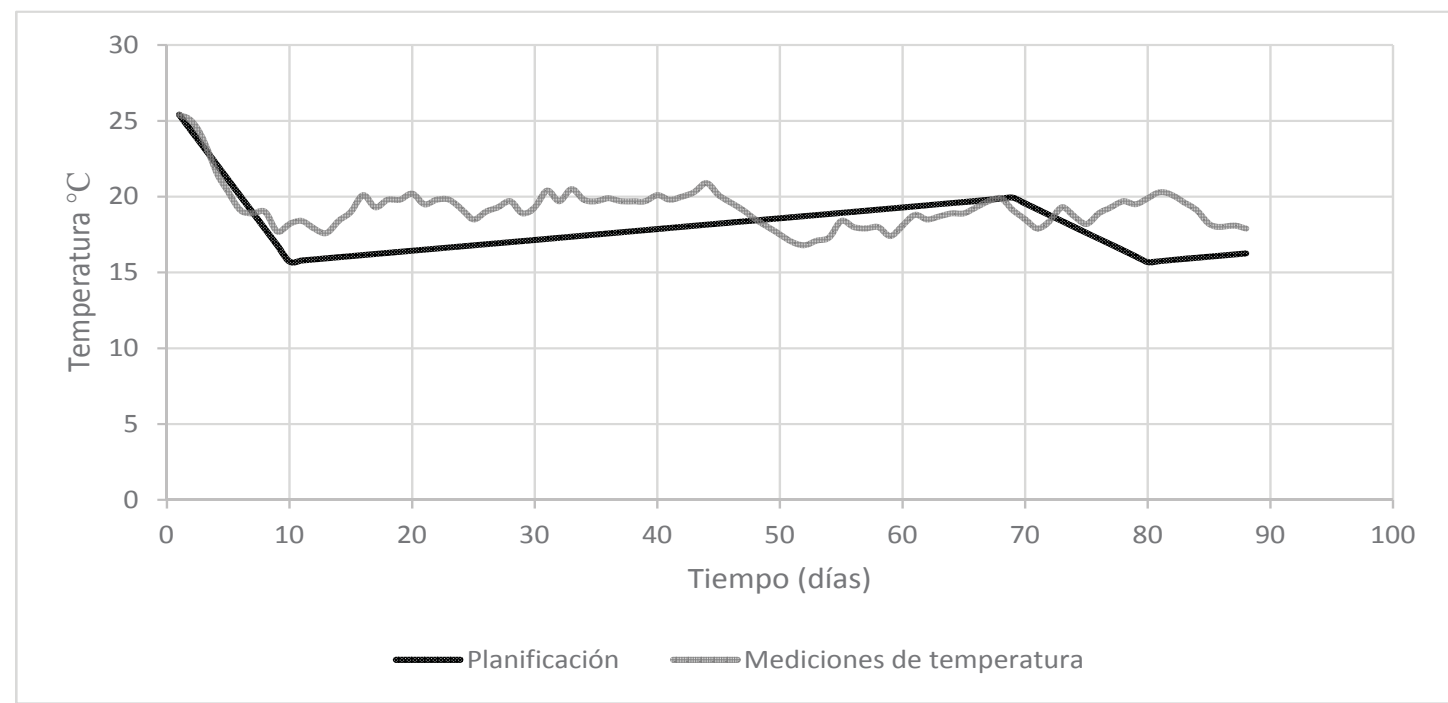

FIGURA 7 - PLANIFICACIÓN DE LA CONEXIÓN DE LOS CARROS DE FRÍO EN EL SMR

Es importante monitorear las plagas dentro de los SMR, si se logra controlar la temperatura correctamente, para establecer parámetros de clima más apropiados para el almacenamiento del maíz en el caso de que los actuales no resuelvan el problema.

\section{CONCLUSIÓN}

La gestión del almacenamiento de granos, en particular el maíz, es muy importante en la proyección estratégica de cualquier país para asegurar la alimentación de sus habitantes. La utilización de tecnologías, como los Silos Metálicos Refrigerados, permite aumentar el tiempo de almacenamiento y conservación de granos, manteniendo las plagas poscosecha en límites que permita su utilización para consumo humano. En la provincia Cienfuegos se vendieron miles de toneladas de maíz para consumo animal, teniendo este como destino inicial el consumo humano debido a niveles altos de plagas.

Para evitar la infestación del grano es necesario que la temperatura y la humedad en el SMR se encuentren en control, este proceso está a cargo de la correcta conexión de los carros de frío. En el análisis del comportamiento de dichas variables se detectaron problemas en la temperatura, el proceso de enfriamiento se encuentra descentrado con valores por encima del límite superior de especificaciones $\left(20^{\circ} \mathrm{C}\right)$.

Se pronosticó el comportamiento de la temperatura utilizando las líneas de tendencia, el comportamiento de los errores del modelo de pronóstico muestran un MAD de 0,737 muy bueno por debajo de $1{ }^{\circ} \mathrm{C}$ y una señal de rastreo de $-10,973$. A pesar de que esta última medida de error no tiene un comportamiento adecuado, puede ser utilizado para la realización de la planificación debido a que predice por exceso en la mayoría de las ocasiones lo que no pone en riesgo la utilidad del mismo pues solamente provocaría una planificación un poco adelantada.

La utilización de este modelo de pronóstico permite la planificación acertada de la conexión del carro de frío en el SMR para lograr mantener la temperatura por dentro de los límites establecidos. También puede contribuir a la programación de acciones de mantenimiento de la tecnología, y balancear la disponibilidad de los mismos con las necesidades en los SMR de la región. 


\section{ABSTRACT \\ PLANNIG OF COOLING PROCESS IN REFRIGERATED METALLIC GRAINHOUSES FOR CORN STORAGE}

The present research aims to forecast the temperature behavior in a Refrigerated Metallic Grainhouse (RMG) to carry out an efficient cooling process plan. This process occurs by connecting cold air compressors attached to vehicles and shared among several RMG. Climate variations during storage were analyzed statistically using the capability analysis technique, which has shown that temperature was not in accordance with the specifications for corn storage. This problem affects the grain quality due to postharvest plagues. A forecasting temperature model was developed using trend lines, and validated through the error analysis. The model was applied to the cooling process planning for corn storage which grants proper control of the correct temperature in the RMG.

KEY-WORDS: CORN STORAGE; TEMPERATURE FORECAST; POST-HARVEST PLAGUE CONTROL.

\section{REFERENCIAS}

1 ABRAHAM, B.; LEDOLTER, J. Statistical methods for forecasting. New Jersey: John Wiley, 2009.

2 ACOSTA, R. Selección participativa de germoplasma cubano de maíz (Zea mays, L.) en el sistema local de Batabanó, la Habana. Instituto Nacional de Ciencias Agrícolas, v. 28, n. 2, p. 63-70, 2012.

3 BERRY, S.T.; ROBERTS, M.J.; SCHLENKER, W. Corn production shocks in $\mathbf{2 0 1 2}$ and beyond: implications for food price volatility. Cambridge: National Bureau of Economic Research, 2012.

4 BOX, G.E.; JENKINS, G.M.; REINSEL, G.C. Time series analysis: forecasting and control. New Jersey: John Wiley \& Sons, 2013.

5 CHOWDHURY, M.R. Process capability analysis in pharmaceutical production. International Journal of Pharmaceutical and Life Sciences, v.2, n.2, p.85-89, 2013.

6 CHUNHUA, H.Z.L. Temperature control for grain storage in large warehouse in Guangdong Province. Grain Storage, v. 1, p.16-18, 2011.

7 Centro Nacional de Sanidad Vegetal (CNSV). Curso sobre manejo integrado de plagas en almacenes, silos, instalaciones de la industria molinera y transportación de alimentos. La Habana: Impresiones Minag, 2006.

8 DOMINGUEZ-PACHECO, A.; HERNÁNDEZ AGUILAR, C.; ZEPEDA BAUTISTA, R.; MARTÍNEZ ORTIZ, E.; CRUZOREA, A. Análisis térmico de semilla de maíz con plaga por microscopía fotopiroeléctrica. Superficies y Vacío, v.25, n.2, p.92-96, 2012.

9 DOMÍNGUEZ UMPIÉRREZ, J.E.; MARRERO ARTABE, L. Catálogo de la entomofauna asociada a almacenes de alimentos en la Provincia de Matanzas. Fitosanidad, v.14, n.2, p. 75-82, 2010.

10 HUI, J.H.C.D.Y.; LIWEI, L.L.R.F.B.; JIAMIN, J.X.S. Grain storage by temperature control in large warehouses with dynamic heat insulation structure. Grain Storage, v. 2, p.12-22, 2010.

$11 \mathrm{HUI}$, Z.H.W. Investigation and analysis on temperature control test for grain storage by air conditioner in Fujian Area. Grain Storage, v.5, p.74-82, 2010.

12 KIM, T.; DEKKER, R.; HEIJ, C. The impact of forecasting errors on warehouse labor efficiency: a case study in consumer electronics. Rotherdam: Erasmus School of Economics (ESE), 2013.

13 LADINO GAIBOR, Á.E. Estudio del manejo postcosecha del maíz tierno (Zea mays L.) procedente del Cantón San Miguel de la Provincia de Bolívar. 2012. 106 f. Disertación (Ingeniero Agro Industrial), Escuela Politécnica Nacional, Quito, 2012.

14 LANDIS, D.A.; GARDINER, M.M.; VAN DER WERF, W.; SWINTON, S.M. Increasing corn for biofuel production reduces biocontrol services in agricultural landscapes. Proceedings of the National Academy of Sciences, v.105, n.51, p.2055220557, 2008.

15 LEI, Y. Application and prospect of advanced technology for grain storage. Grain Storage, v. 6, p. 23-28, 2011.

16 MICROSOFT CORPORATION. Ms Excel. 2007. Disponible en: <http://office.microsoft.com/en-ca/excel/> Visitado el: 12 de enero de 2012.

17 MONTGOMERY, D.C.; RUNGER, G.C.; HUBELE, N.F. Engineering statistics. New Jersey: John Wiley \& Sons, 2009.

18 PALIWAL, R.L.; GRANADOS, G.; LAFITTE, H.R.; VIOLIC, A.D.; MARATHÉE, J.-P. El maíz en los trópicos: mejoramiento y producción. Roma: FAO, 2001. 
19 PINGALI, P.L. Cimmyt 1999-2000 World Maize Facts and Trends - Meeting world maize needs: technological opportunities and priorities for the public sector. México, DF: Centro Internacional de Mejoramiento de Maiz y Trigo, 2001.

20 RUIZ ARBOLEDA, L.E. Plan de mejoramiento del proceso de manejo poscosecha y de las operaciones de los sistemas de secado de maíz para el sector agrícola en el Cantón Ventanas, en la Provincia De Los Ríos. 2010.83 f. Disertación (Ingeniero Agro Industrial), Escuela Politécnica Nacional, Quito, 2010.

21 STATPOINT TECHNOLOGIES. Statgraphics Centurion, Xvi. 2009. Disponible en: <www.statgraphics.com> Visitado el: 12 de enero de 2012.

22 WEI, H.M.H.X.Z.; JIBIN, C. Field maize storage by using technology of nitrogen filling and oxygen lowering by pressure swing adsorption (Psa). Grain Storage, v.2, p.7-18, 2011.

23 WEIZHI, X. Maize safe storage technology in silos in Southern Coastal Areas. Grain Storage, v.4, p. 6-15, 2010.

24 YANG, S.L.; SHEN, J.; LI, Z.M.; TAN, B.X.; ZHAO, K. Temperature and humidity measurement system for grain storage based on Nrf 905. Advanced Materials Research, v.383, p.5012-5016, 2012. 